\title{
Protein Transfection of Intact Microvessels Specifically Modulates Vasoreactivity and Permeability
}

\author{
John H. Tinsley David C. Zawieja Mack H. Wu Elena E. Ustinova \\ Wenjuan Xu Sarah Y. Yuan
}

Departments of Surgery and Medical Physiology, Cardiovascular Research Institute, Texas A\&M University

System Health Science Center, Temple, Tex., USA

\section{Key Words}

Permeability · Transfection · PKC · Nitric oxide synthase • Vasodilation

\begin{abstract}
Precise regulation of microvascular tone and barrier function is essential for proper coronary perfusion and performance. Agonist-induced alterations in either or both of these functions ultimately lead to microcirculatory dysfunction and cardiac insufficiency. Two important pathways involved in regulating vasomotor response and barrier function are the activation of nitric oxide synthase (NOS) and upregulation of protein kinase C (PKC). To date, studies of these two signaling proteins have relied mainly on pharmacological approaches. Unfortunately, the specificity of various inhibitors can be cause for concern. In order to address this problem, a protein transfection technique we developed for cultured endothelial cells has been modified and applied to isolated, intact coronary microvessels. Our results from green fluorescent protein transfection in arterioles and venules showed that this procedure could be used to introduce proteins into the microvascular wall. By transfecting inhibitor peptides against NOS and PKC into coronary arterioles and venules, we have been able to determine the specific roles of these two enzymes in vasodilation and hyperpermeability responses.
\end{abstract}

Copyright $\odot 2001$ S. Karger AG, Basel

\section{Introduction}

Microvascular endothelium, an intricate cell lining between the bloodstream and tissues, participates in homeostatic mechanisms, including antithrombogenesis, angiogenesis, regulation of leukocyte dynamics, and control of local tissue perfusion. Another important function of capillary and venular endothelium is to form a semipermeable barrier that governs the transvascular movement of fluid and solutes [1, 2]. Binding of certain agonists to endothelial cells elicits intracellular signaling events that alter vasomotor function and/or barrier property of microvessels [3-7]. These processes are involved in the physiological and pathophysiological regulation of local blood flow and blood-tissue exchange.

Coronary arterioles possess autoregulatory function. These vessels are capable of constricting or dilating in response to changes in intravascular pressure and flow, as well as to the accumulation of metabolites [8]. Recent studies have determined that nitric oxide (NO) plays a critical role in the regulation of autoregulatory function [9-11]. The study of NO has relied upon pharmacological manipulation of nitric oxide synthase (NOS). Questions are often raised as to the specificity and selectivity of certain pharmacological agents. A more precise method of activating or inhibiting particular enzymes would allow for greater confidence in future experiments.

Dr. John H. Tinsley

Department of Medical Physiology, Texas A\&M University System Health Science Center 702 SW HK Dodgen Loop, Room 206F

Temple, TX 76504 (USA)

Tel. +1 254742 7038, Fax +1 254742 7145, E-Mail jht@tamu.edu 
The role of protein kinase $\mathrm{C}$ (PKC) in the mediation of microvascular hyperpermeability has been widely documented. Activation and inhibition of PKC generally results in increases and decreases, respectively, in permeability [3, 12-14]. However, once again the absolute specificity of pharmacological agents may be questioned. Furthermore, the majority of this work has been performed either with the tracer clearance technique in vivo, where the global influence of hemodynamics is difficult to control, or through measurement of transendothelial flux of macromolecules in cultured cell monolayers, which is not reflective of the physiological conditions in the intact vessel. We were able to minimize these problems in previous studies using isolated, intact coronary microvessels [3, $15]$.

In order to expand our experimental capabilities using the intact microvessel technique, we first developed a monolayer permeability model using coronary venular endothelial cells (CVECs) [16, 17]. Additionally, we sought a procedure that would allow us to control the function of cellular proteins with greater specificity than pharmacological agents can offer. To accomplish this, a protocol for transfection of otherwise cell-impermeable proteins and peptides was developed using CVECs [17, 18]. Others have successfully used this technique to transfect protein into skeletal muscle resistance arteries [19]. The current study describes the application of the protein transfection technique to intact coronary microvessels, where we can now study the physiological roles of specific proteins via their inhibition using specific peptide inhibitors. Not only does this minimize the possible non-specificity of pharmacological agents but also allows for immediate studies following transfection, avoiding the lag time associated with transcription and translation in the case of DNA transfection.

Our results begin by demonstrating the ability of the transfection technique to successfully introduce green fluorescent protein (GFP) into the wall of intact coronary venules and arterioles. This is followed by experiments that for the first time selectively inhibit either PKC or endothelial NOS (eNOS) activity in venules and arterioles, respectively, using inhibitory peptides. In the case of PKC, the inhibitor peptide specifically binds to the catalytic domain of the enzyme, blocking autophosphorylation and PKC substrate phosphorylation. PKC peptide inhibition significantly attenuated the hyperpermeability response in venules elicited by the PKC activator phorbol 12-myristate 13-acetate (PMA), but the hyperpermeability response to the $\mathrm{PKC}$-independent factor histamine was not changed in transfected venules. Additionally, eNOS peptide inhibition significantly reduced the vasodilatory response to the NO- and endothelium-dependent vasodilators bradykinin and serotonin in intact arterioles. This eNOS peptide inhibition had no effect on the vasodilatory effect of the endothelium-independent, smooth muscledependent vasodilator sodium nitroprusside (SNP). The specificity and the inhibiting capacity of the transfected peptides are further supported by in vitro studies showing a dramatically attenuated response of enzyme activity and NO production in peptide-transfected endothelial cells upon agonist stimulation.

\section{Materials and Methods}

\section{Chemicals and Drugs}

The chemicals used were phorbol 12-myristate 13-acetate (PMA) (Calbiochem, San Diego, Calif.), green fluorescent protein (GFP) (Roche, Indianapolis, Ind.), and bradykinin, serotonin, sodium nitroprusside (SNP), LNMMA, and FITC/albumin (Sigma, St. Louis, Mo.).

\section{Isolation and Perfusion of Microvessels}

Pigs weighing 9-13 kg were sedated with ketamine $(2.5 \mathrm{mg} / \mathrm{kg}$ i.m.) and rompun $(2.25 \mathrm{mg} / \mathrm{kg}$ i.m.), anesthetized with pentobarbital sodium $(25 \mathrm{mg} / \mathrm{kg}$ i.v.), and treated with heparin $(250 \mathrm{U} / \mathrm{kg}$ i.v. $)$. After a tracheotomy, intubation, and ventilation, a left thoracotomy was performed. The heart was electrically fibrillated, excised, and placed in $4^{\circ} \mathrm{C}$ physiological saline. The left descending artery was cannulated and $3 \mathrm{ml}$ of india ink-gelatin-physiological salt solution were infused to clearly define microvessels.

The methods for isolating and cannulating coronary microvessels have been described in our previous studies [6, 7, 15, 20, 21]. Coronary arterioles $(1.0-1.5 \mathrm{~mm}$, diameter $40-80 \mu \mathrm{m})$ and venules $(0.8-$ $1.2 \mathrm{~mm}$, diameter $20-50 \mu \mathrm{m}$ ) were dissected from surrounding myocardium in a dissecting chamber containing an albumin-physiological salt solution (APSS) at $4{ }^{\circ} \mathrm{C}$ with the aid of an SV 11 stereo dissecting microscope (Carl Zeiss, Thomwood, N.Y.). APSS consists of (in mmol/l): $145.0 \mathrm{NaCl}, 4.7 \mathrm{KCl}, 2.0 \mathrm{CaCl}_{2}, 1.17 \mathrm{MgSO}_{4}, 1.2$ $\mathrm{NaH}_{2} \mathrm{PO}_{4}, 5.0$ glucose, 2.0 pyruvate, 0.02 EDTA, and 3.0 3-(N-morpholino)propanesulfonic acid buffer. The vessel was transferred to a cannulating chamber that was mounted on a Zeiss Axiovert 135 inverted microscope. The isolated vessel was cannulated with a micropipette on each end and secured with 11-0 suture (Alcon, Fort Worth, Tex.). A third smaller pipette was inserted into the inflow pipette. The vessel was perfused with either APSS through the outer inflow pipette or APSS containing FITC-albumin through the inner inflow pipette. Each cannulating micropipette was connected to a reservoir so that the intraluminal pressure and flow velocity could be adjusted independently by simultaneously changing the height of inflow and outflow reservoirs in equal magnitude. A transillumination microscopic picture of a cannulated microvessel has been presented in a previous study [20]. The bath solution in the chamber was maintained at $37^{\circ} \mathrm{C}$ and $\mathrm{pH} 7.4$ throughout the experiments. The image of vessels was projected onto a Hamamatsu charge-coupled device-intensified camera and was displayed on a high-resolution monochromatic video monitor and recorded onto a VHS video 
recorder. Vessel diameter was measured on-line with a video caliper, and the intraluminal flow velocity was measured with an optical Doppler velocimeter (Microcirculation Research Institute, Texas A\&M University, College Station, Tex.).

\section{Measurement of Venular Permeability}

The permeability of the vessel was measured with a fluorescence rationing technique described previously $[6,7,15,20,21]$. Using an optical window of a video photometer positioned over the venule and adjacent space on the monitor, the fluorescent intensity from the window was measured and digitized on-line by a Power Macintosh computer. In each measurement, the isolated venule was first perfused with APSS through the outer inflow pipette to establish baseline intensity. The venular lumen was then rapidly filled with fluorochromes by switching the perfusion to the inner inflow pipette that contained FITC-albumin. This produced an initial step-increase, followed by a gradual increase, in the intensity of fluorescence. There was a step-decrease of intensity when the fluorescent-labeled molecules were washed out of the vessel lumen by switching the perfusion back to the outer inflow pipette. The apparent solute permeability coefficient of albumin $(\mathrm{Pa})$ was calculated using the equation $\mathrm{Pa}=$ $\left(1 / \Delta \mathrm{I}_{\mathrm{f}}\right)\left(\mathrm{dI}_{\mathrm{f}} / \mathrm{dt}\right)_{0}(\mathrm{r} / 2)$, where $\Delta \mathrm{I}_{\mathrm{f}}$ is the initial step increase in fluorescent intensity, $\left(\mathrm{dI}_{\mathrm{f}} / \mathrm{dt}\right)_{0}$ is the initial rate of gradual increase in intensity as solutes diffuse out of the vessel into the extravascular space, and $r$ is the venular radius.

\section{Protein Transfection of Isolated Microvessels}

To transfect venules and arterioles with GFP, vessels were perfused for $1 \mathrm{~h}$ with GFP at $10 \mu \mathrm{g} / \mathrm{ml}$ in the presence of the polyamine transfection reagent TransIT-LT1 (PanVera, Madison, Wisc.) at $10 \mu \mathrm{l} / \mathrm{ml}$. Vessels were fixed and scanned at $2-\mu \mathrm{m}$ increments using a Meridian ULTIMA Z-laser confocal microscope system (Genomic Solutions, Lansing, Mich.) equipped with fluorescence filters (excitation $=400 \mathrm{~nm}$ and emission $=509 \mathrm{~nm}$ ).

To study the specific effect of PKC-inhibiting peptide on microvascular permeability, venules were perfused at a constant pressure gradient of $20 \mathrm{~cm} \mathrm{H}_{2} \mathrm{O}$ and a flow velocity of $7 \mathrm{~mm} / \mathrm{s}$. The perfusate contained either the TransIT-LT1 at $10 \mu \mathrm{l} / \mathrm{ml}$ or PKC-inhibitory peptide (N-Arg-Phe-Ala-Arg-Lys-Gly-Ala-Leu-Arg-Gln-Lys-Asn-ValHis-Glu-Val-Lys-Asn-C; MW $=2,149$ ) at $10 \mu \mathrm{g} / \mathrm{ml}$ or both. This peptide is a pseudosubstrate of PKC that inhibits both autophosphorylation and protein substrate phosphorylation [22]. For a nonspecific PKC peptide, the above inhibitory peptide sequence was scrambled to: N-Arg-His-Val-Lys-Phe-Ala-Lys-Arg-Val-Asn-Ala-Arg-Gly-AsnGln-Lys-Glu-Leu-C; MW = 2,152. Following a transfection period of $1 \mathrm{~h}$, PMA was added to the suffusion bath in a concentration of $10^{-5}$ $\mathrm{mol} / \mathrm{l}$, an optimal dosage that has been shown to induce a submaximal increase in venular permeability $[3,21]$. The effect of PMA on $\mathrm{Pa}$ was examined after $15 \mathrm{~min}$. After PMA was cleared, histamine $\left(10^{-5} \mathrm{~mol} / \mathrm{l}\right)$ was added to examine the responsiveness of the venules to PKC-independent hyperpermeability factors.

To examine the effect of eNOS-inhibiting peptide on the vasodilatory responsiveness, coronary arterioles were isolated and perfused at a constant intraluminal pressure of $70 \mathrm{~cm} \mathrm{H}_{2} \mathrm{O}$ without flow. The vessel was perfused for $1 \mathrm{~h}$ with TransIT-LT1 $(10 \mu \mathrm{l} / \mathrm{ml})$ either by itself or in combination with an eNOS blocking peptide (N-Pro-TyrAsn-Ser-Ser-Pro-Arg-Pro-Glu-Gln-His-Lys-Ser-Tyr-Lys-Cys-C; MW $=1,962)$ at $10 \mu \mathrm{g} / \mathrm{ml}$. This peptide is reported by Calbiochem to specifically inhibit eNOS activity. For a nonspecific eNOS peptide, the above inhibitor peptide sequence was scrambled to: N-Pro-Tyr-Pro-
Ser-Cys-Tyr-GluLys-His-Pro-Ser-Asn-Lys-Gln-Ser-Arg-C; $\mathrm{MW}=$ 1,921. After preconstriction with $\mathrm{KCl}(100 \mathrm{mmol} / \mathrm{l})$, a dose response of bradykinin- $\left(10^{-5}-10^{-9} \mathrm{~mol} / \mathrm{l}\right)$ or serotonin- $\left(10^{-5}-10^{-9} \mathrm{~mol} / \mathrm{l}\right)$ induced changes in arteriolar diameter was measured.

\section{NO Assay in Coronary Venular Endothelial Cell Monolayers}

CVECs were grown to confluence in gelatin-coated $35-\mathrm{mm}$ dishes. Cells were transfected with TransIT-LT1 $(10 \mu \mathrm{l} / \mathrm{ml})$ and eNOS-blocking peptide $(10 \mu \mathrm{g} / \mathrm{ml})$ for $1 \mathrm{~h}$ or treated with LNMMA $\left(10^{-4} \mathrm{~mol} / \mathrm{l}\right)$ for $15 \mathrm{~min}$. Using a trypan blue exclusion assay, our previous study determined that the survival rate of CVECs transfected under these conditions is $97.73 \%$ vs. $97.92 \%$ for non-transfected cells [18]. Following two washes and a medium change, bradykinin or serotonin was added to the cells at $10^{-5} \mathrm{~mol} / \mathrm{l}$. Medium was then assayed for NO levels after 5 min incubation using a kit from Calbiochem (Catalog 482655) according to their protocol. Background NO levels from unused media were subtracted from experimental values.

\section{Data Analysis}

The microvascular parameters were measured 2-3 times for each vessel at each experimental intervention and the values were averaged. For each experimental condition, the changes in the diameter and the permeability coefficient from different vessels were normalized to the control values and were reported as mean \pm SE. For cell studies, at least three measurements were made in separate dishes of cells under each intervention. For all experiments, $n$ is given as the number of vessels or dishes of cells studied. ANOVA was used to evaluate the significance of intergroup differences. A value of $p<$ 0.05 was considered significant for the comparisons.

\section{Results}

\section{Confocal Microscopic Observation of GFP \\ Transfection}

In order to determine the efficacy of protein transfection to isolated, intact microvessels, we utilized GFP in conjunction with confocal microscopy. Venular transfection is shown in figure 1, panels V1-V6. Images were captured at $2-\mu \mathrm{m}$ increments moving from near the vessel surface toward the lumen. As indicated in our previous studies [20], coronary venules of this size have very little continuous smooth muscle cells surrounding the endothelium. The fluorescent images show GFP situated in a longitudinal pattern through the vessel, consistent with the known typical pattern of endothelial cells. In figure 1, panels A1-A6, we show GFP fluorescence staining after transfection into an arteriole. Figure 1, panel A2 clearly shows a longitudinal, spiral pattern of GFP protein. In figure 1, panels A3-A6, the lumen becomes visible and the majority of the GFP fluorescence is found at the edges of this lumen. It should be pointed out that non-transfected control vessels and vessels treated with TransITLT1 only did not exhibit fluorescence. 

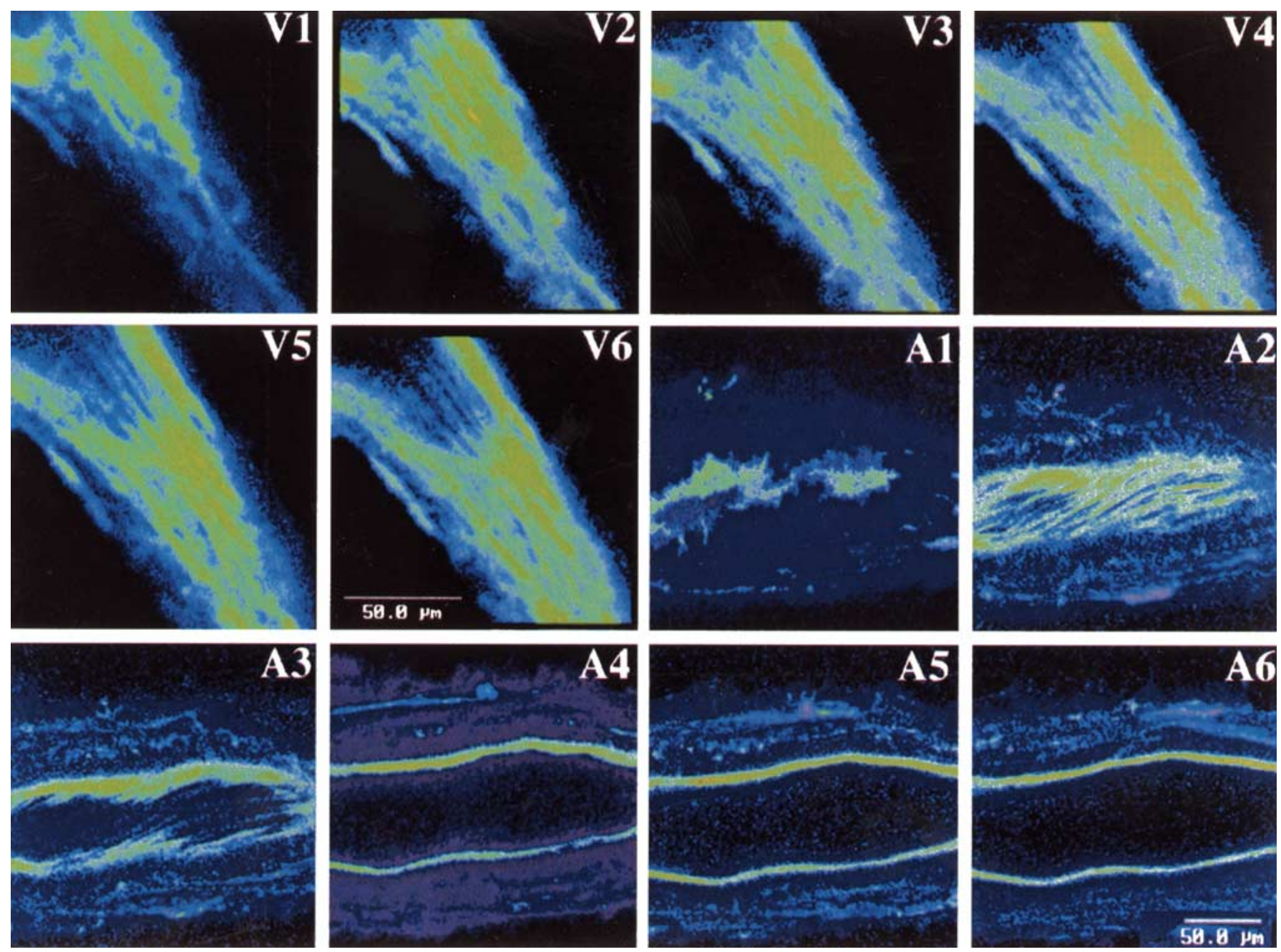

Fig. 1. Green fluorescent protein (GFP) transfection in coronary venules and arterioles. Venules (V1-V6) and arterioles (A1-A6) were transfected with GFP for $1 \mathrm{~h}$, fixed, and scanned at 2- $\mu \mathrm{m}$ increments using confocal microscopy. In both venules and arterioles, the GFP pattern is longitudinal along the axis of the vessel, consistent with the pattern formed by endothelial cells.

\section{PKC-Inhibitory Peptide Transfection into Venules}

Previous studies on both cultured endothelial cells and intact coronary venules have shown that PMA-induced increases in PKC activity increase permeability of the endothelium [3, 16]. This hyperpermeability response was examined more directly by both inducing PKC activation with PMA and then decreasing this activity using a PKC-inhibitory peptide, which we had previously shown to lower PKC activity in CVECs [18]. Results depicted in figure 2 show permeability increases in response to PMA in venules treated with TransIT-LT1 only (column C) and peptide only (column D) similar to the response seen with PMA only (column B). This demonstrates that the transfection reagent or peptide alone does not affect the venu- lar response to PMA. In contrast, when the PKC-inhibitory peptide is applied to the venules in combination with TransIT-LT1, no significant increases in permeability are observed after PMA exposure (fig. 2, column E). However, no attenuation of PMA-induced hyperpermeability is seen when a scrambled peptide with the same amino acid content is transfected (fig. 2, column F), demonstrating that inhibitory effects are sequence-specific. Furthermore, histamine-induced increases in venular permeability were well preserved after transfection of the PKCinhibiting peptide (fig. 2, column G). The magnitude of this post-transfection histamine-induced hyperpermeability is consistent with our previous studies [7]. Because histamine is known to cause venular hyperpermeability via a 


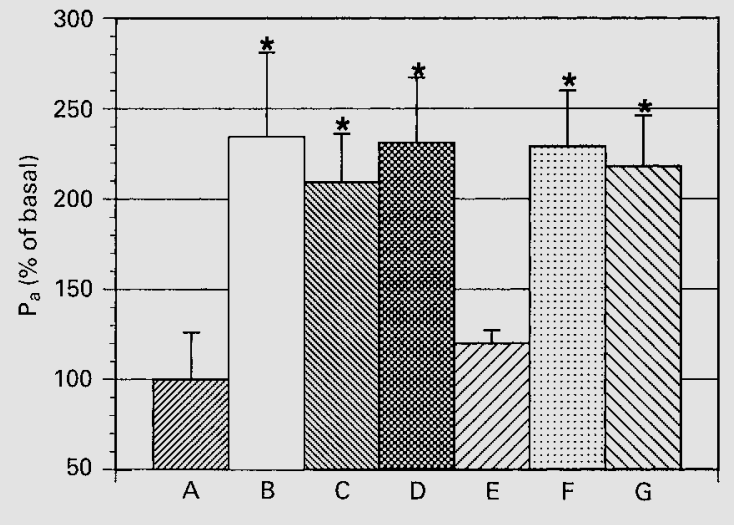

Fig. 2. $P K C$-inhibitory peptide transfection in coronary venules. Venules were perfused with physiological salt solution containing TransIT-LT1 (10 $\mu \mathrm{l} / \mathrm{ml})(\mathrm{C})$; PKC-inhibitory peptide (D); TransITLT1/inhibitory peptide (E, G); or TransIT-LT1/nonspecific peptide (F). After $1 \mathrm{~h}$, PMA was added at $10^{-5} \mathrm{~mol} / \mathrm{l}$ to B, C, D, E, and F. Permeability measurements were taken $15 \mathrm{~min}$ after PMA and expressed as percentage of basal (A). Histamine was added at $10^{-5} \mathrm{~mol} / \mathrm{l}$ at the end of the experiment to confirm the inflammatory responsiveness of the vessels $(\mathrm{G})$. For each treatment, $\mathrm{n}=5$. * indicates significant increase in basal permeability, $\mathrm{p}<0.05$.

PKC-independent mechanism, the results further confirm the efficacy and specificity of peptide transfection. It should be noted that neither the transfection reagent nor PKC-inhibiting peptide alone altered basal $\mathrm{Pa}$.

\section{NO Levels in CVECs Transfected with}

\section{eNOS-Inhibiting Peptide}

Knowing that we could successfully transfect proteins into the luminal wall of microvessels, we next wanted to target an endothelial-specific enzyme. We examined this in a preliminary fashion by transfecting CVEC monolayers with a peptide that was suspected to inhibit eNOS activity, treating with either bradykinin or serotonin, and measuring NO levels in the medium after $5 \mathrm{~min}$. As figure 3 shows, bradykinin and serotonin increased basal NO levels in cellular medium 8-10 fold after $5 \mathrm{~min}$. However, when CVECs are transfected for $1 \mathrm{~h}$ with the eNOSinhibiting peptide, bradykinin- and serotonin-induced increases in NO levels are completely attenuated (fig. 3). This attenuation is also observed when the cells are treated with LNMMA prior to bradykinin or serotonin exposure (fig. 3).

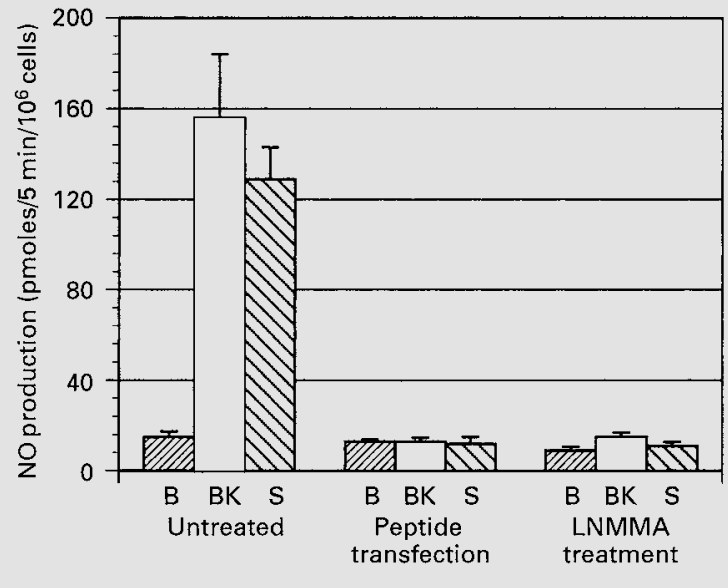

Fig. 3. NO levels in cultured coronary venular endothelial cells (CVECs) transfected with eNOS-inhibitory peptide. CVECs were left untreated; transfected with TransIT-LT1 $(10 \mu \mathrm{l} / \mathrm{ml}) / \mathrm{eNOS}$-inhibitory peptide $(10 \mu \mathrm{g} / \mathrm{ml})$; or treated with LNMMA $\left(10^{-4} \mathrm{~mol} / \mathrm{l}\right)$. One hour after transfection or 15 min after LNMMA, cells were treated with bradykinin $\left(10^{-5} \mathrm{~mol} / \mathrm{l}\right)$ or serotonin $\left(10^{-5} \mathrm{~mol} / \mathrm{l}\right)$. NO production was measured $5 \mathrm{~min}$ after bradykinin or serotonin treatment and reported as picomoles per $10^{6}$ cells. $\mathrm{B}=$ basal, $\mathrm{BK}=$ bradykinin, $\mathrm{S}=$ serotonin. In the untreated group, bradykinin and serotonin induced a significant increase in $[\mathrm{NO}], \mathrm{p}<0.05$. There were no significant differences in [NO] between peptide-transfected and LNMMAtreated cells. For each treatment, $n=4$.

\section{Inhibition of eNOS-Induced Arteriolar Dilation}

Serotonin and bradykinin are two dilatory agonists whose action is thought to be dependent on eNOS. Because the cell monolayer data confirmed that we could decrease eNOS activity by transfecting an inhibitory peptide, we wanted to determine the effect of this peptide on intact arterioles exposed to serotonin and bradykinin. In figure $4 \mathrm{a}$, we see that arterioles dilate in response to serotonin in a dosage-dependent fashion both before and after exposure to TransIT-LT1 alone, confirming that the transfection reagent itself does not inhibit vessel vasodilatory response. After transfecting an eNOS-inhibitory peptide, the dilatory response to serotonin is greatly attenuated (fig. 4b). When a scrambled peptide with the same amino acid content as the eNOS-inhibitory peptide is transfected, no attenuation of serotonin-induced increases in arteriolar diameter is observed (fig. 4b), confirming that the specific peptide sequence is essential for eNOS inhibition. Arterioles also dilated in response to bradykinin in a dosage-dependent fashion both before and after exposure to TransIT-LT1 (fig. 5a). Again, the specific eNOS-inhibitory peptide attenuated the bradykinin-induced dilation 

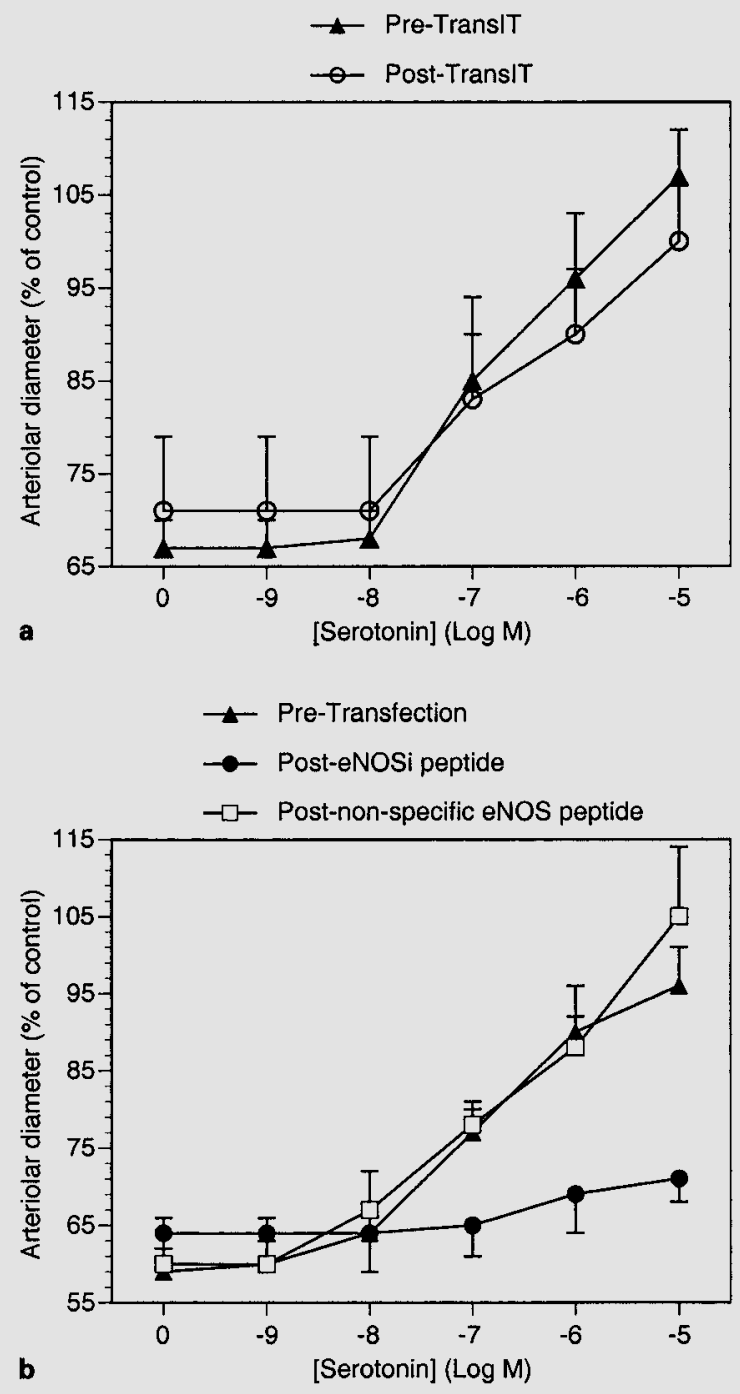

Fig. 4. Serotonin-induced changes in diameter of coronary arterioles transfected with eNOS-inhibitory peptide or nonspecific peptide. Panel a represents 5 different arterioles whose diameters were measured before and after serotonin in the absence of TransIT-LT1 $(10 \mu \mathrm{l} / \mathrm{ml})$ (Pre-TransIT). The same vessels were then exposed to TransIT-LT1 for $1 \mathrm{~h}$ followed by serotonin (Post-TransIT). Panel b also represents 5 different arterioles whose diameters were measured before and after serotonin in the absence of TransIT-LT1 (Pre-Transfection). Vessels were transfected with TransIT-LT1 and eNOSinhibitory peptide $(10 \mu \mathrm{g} / \mathrm{ml})$ (Post-eNOSi peptide) or TransIT-LT1/ nonspecific peptide $(10 \mu \mathrm{g} / \mathrm{ml})$ (Post-nonspecific eNOS peptide) for $1 \mathrm{~h}$ followed by serotonin. Serotonin induced significant increases $(\mathrm{p}<0.05)$ in diameter at $10^{-7}, 10^{-6}$, and $10^{-5} \mathrm{~mol} / \mathrm{l}$ (with respect to control levels, no serotonin) in the presence of TransIT-LT1 only and TransIT-LT1/nonspecific eNOS peptide. No significant increases in diameter due to serotonin were observed after TransIT-LT1/eNOSinhibitory peptide. For each treatment, $\mathrm{n}=5$. All diameters were measured 5 min after serotonin dosages.
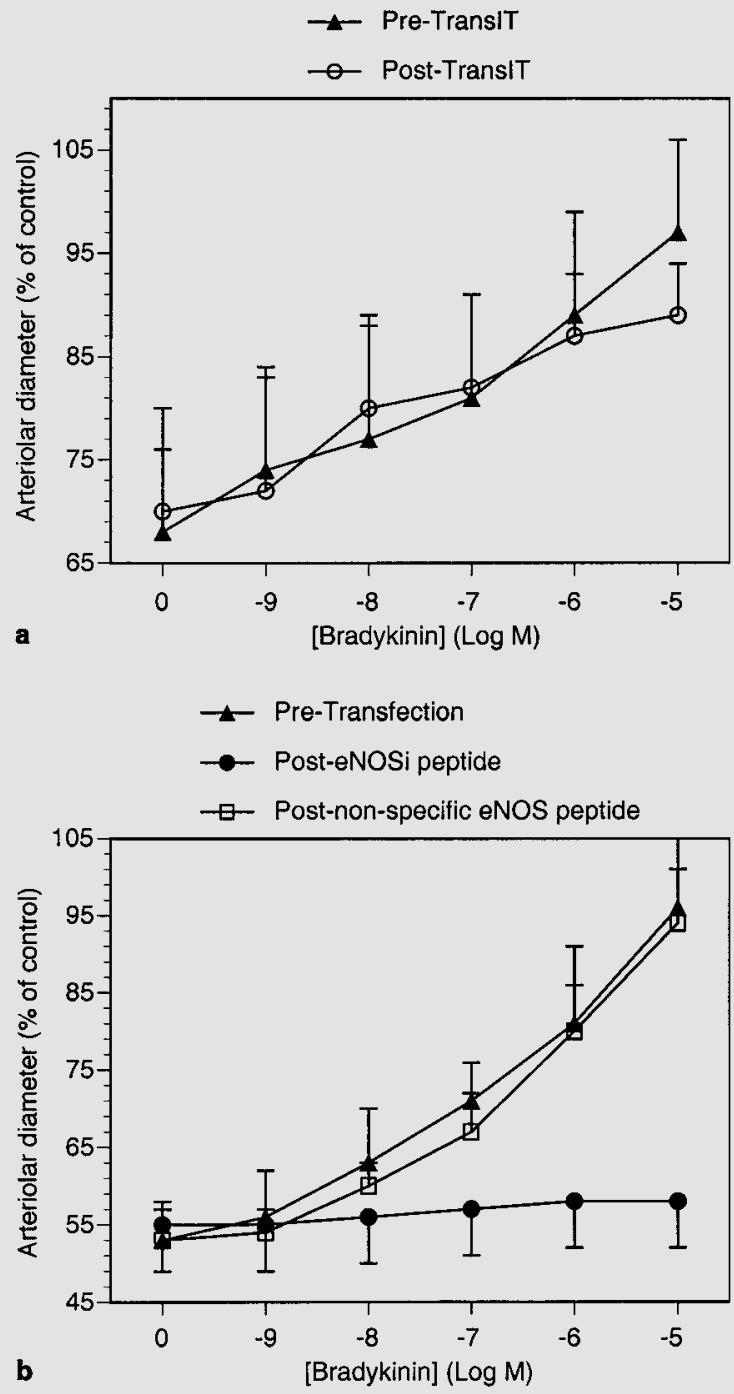

Fig. 5. Bradykinin-induced changes in diameter of coronary arterioles transfected with eNOS-inhibitory peptide or nonspecific peptide. Panel a represents 5 different arterioles whose diameters were measured before and after bradykinin in the absence of TransIT-LT1 $(10 \mu \mathrm{l} / \mathrm{ml})$ (Pre-TransIT). The same vessels were then exposed to TransIT-LT1 for $1 \mathrm{~h}$ followed by bradykinin (Post-TransIT). Panel b also represents 5 different arterioles whose diameters were measured before and after bradykinin in the absence of TransIT-LT1 (PreTransfection). Vessels were transfected with TransIT-LT1 and eNOS-inhibitory peptide $(10 \mu \mathrm{g} / \mathrm{ml})$ (Post-eNOSi peptide) or TransIT-LT1/nonspecific peptide $(10 \mu \mathrm{g} / \mathrm{ml})$ (Post-nonspecific eNOS peptide) for $1 \mathrm{~h}$ followed by bradykinin. Bradykinin induced significant increases $(\mathrm{p}<0.05)$ in diameter at $10^{-7}, 10^{-6}$, and $10^{-5} \mathrm{~mol} / \mathrm{l}$ (with respect to control levels, no bradykinin) in the presence of TransITLT1 only and TransIT-LT1/nonspecific eNOS peptide. No significant increases in diameter due to bradykinin were observed after TransIT-LT1/eNOS-inhibitory peptide. For each treatment, $\mathrm{n}=5$. All diameters were measured 5 min after bradykinin dosages. 


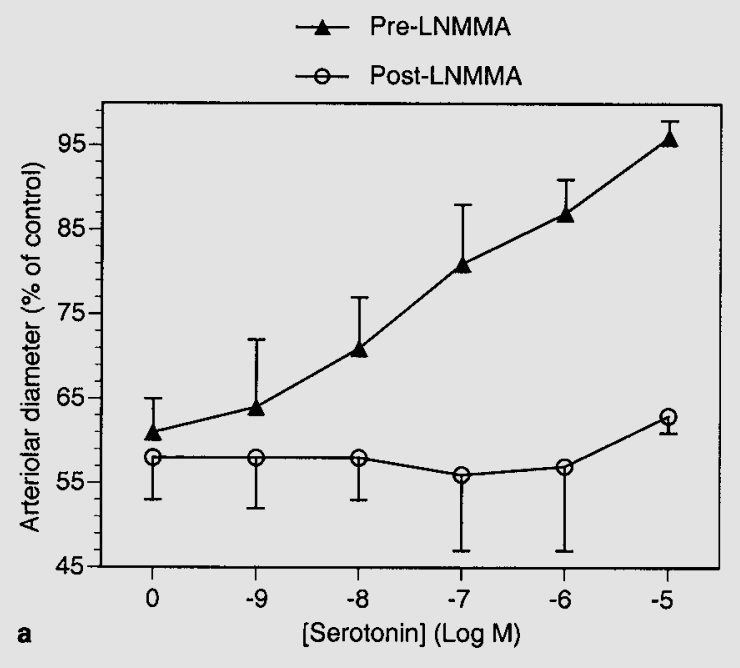

a

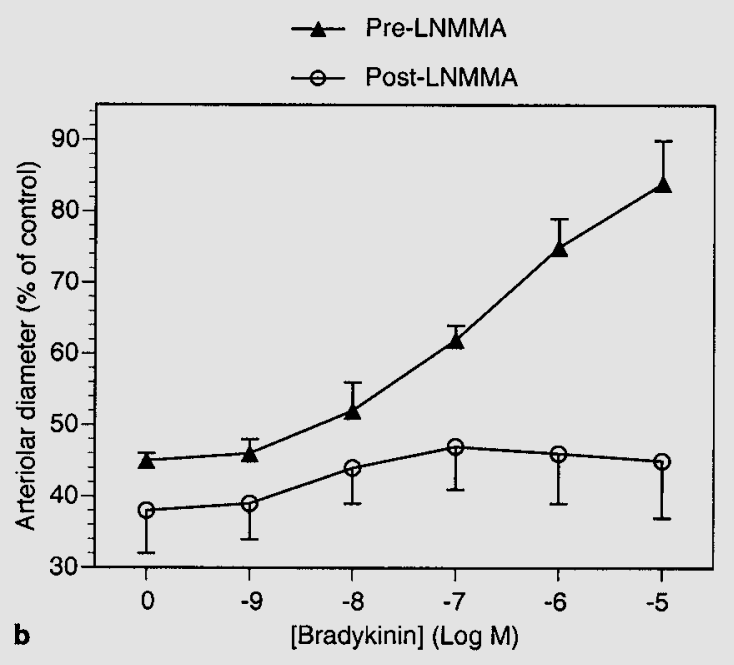

Fig. 6. Serotonin- and bradykinin-induced changes in diameter of coronary arterioles pretreated with LNMMA. Arteriolar diameters were measured before and after LNMMA $\left(10^{-4} \mathrm{~mol} / \mathrm{l}\right)$ in response to serotonin (a) or bradykinin (b). No significant increases due to serotonin or bradykinin treatment were observed when vessels were pretreated with LNMMA. For each treatment, $n=4$. All diameters were measured 5 min after serotonin and bradykinin dosages.

while the scrambled peptide failed to do so (fig. 5b). The widely used NOS inhibitor LNMMA was applied to arterioles in a parallel set of experiments to the eNOS-inhibitory peptide transfection. LNMMA treatment also attenuated both serotonin- and bradykinin-induced dilation in a similar fashion to the peptide transfection (fig. 6a, b).

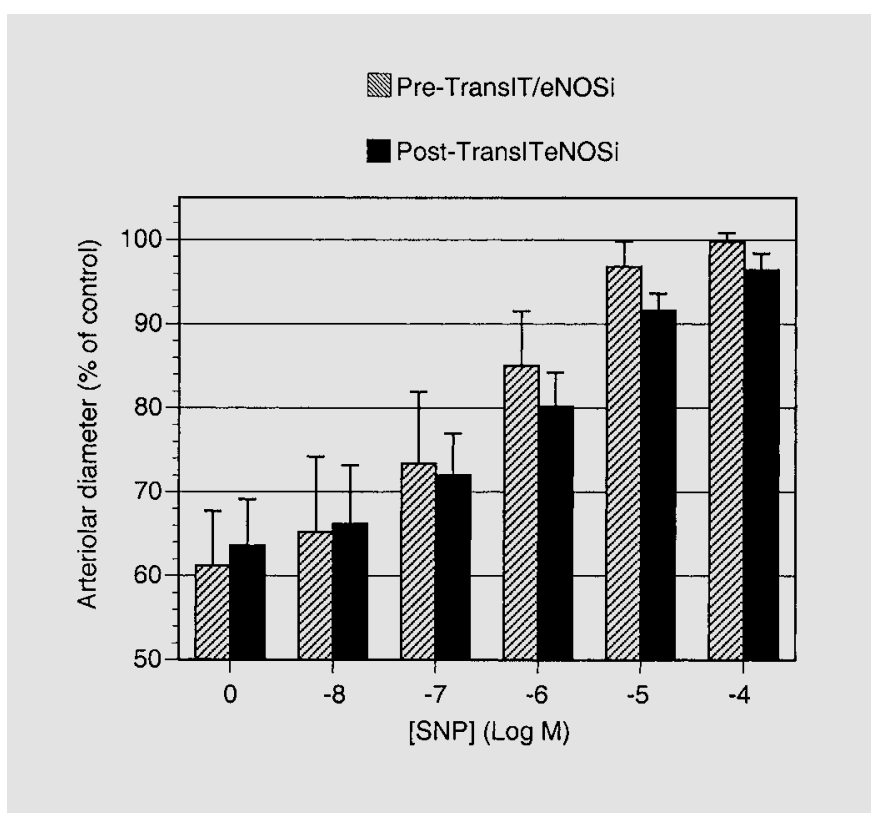

Fig. 7. SNP-induced changes in diameter of coronary arterioles transfected with eNOS-inhibitory peptide. Arteriolar diameters were measured before and after exposure to TransIT-LT1/eNOS-inhibitory peptide for $1 \mathrm{~h}$ in response to SNP. There were no significant differences between the pre-transfected and post-transfected groups at any SNP concentration. For each treatment, $n=4$.

\section{Arteriolar Dilation with Exogenous NO}

The specificity of the eNOS-inhibitory peptide is paramount in the development of this procedure for regulating enzyme function. In order to determine if the peptide transfection altered the cells' ability to respond to increases in NO levels rather than inhibit eNOS activity, we transfected arterioles with the peptide and then treated with SNP, an NO donor. The arterioles responded to the SNP in a dosage-dependent fashion by dilating both before and after eNOS-inhibitory peptide transfection (fig. 7). Peptide transfection did not significantly alter the arteriolar dilation in response to addition of exogenous NO.

\section{Discussion}

Precise regulation of microvascular blood flow and permeability is crucial for the maintenance of coronary perfusion and cardiac function. Coronary arterioles autoregulate themselves by constricting or dilating in response to changes in blood flow or metabolic demand while the endothelium of coronary venules maintains a semiperme- 
able barrier to fluid and macromolecules. Agonist stimulation of arteriolar or venular endothelial cells can lead to intracellular signaling events that modify vasomotor function and/or barrier property of microvessels. Alterations to the delicate balance of coronary blood flow and microvascular permeability elicited by inflammation, ischemiareperfusion injury, atherosclerosis, etc. can lead to coronary insufficiency and myocardial damage. Understanding the molecular basis of arteriole and venule dynamics is crucial to the development of efficient therapeutic and pharmacological strategies combating heart disease.

In vivo observation and quantification of vascular tone and permeability in coronary microvasculature is very difficult due to heart contraction and hemodynamic influences. On the other hand, cultured endothelial cells, which are often derived from large vessels, may not necessarily behave as they would in vivo. Therefore, the ability to isolate intact arterioles and venules has proven to be a more advanced method of examining the physiological processes of vasomotor and barrier function. An important feature of this approach is the ability to directly measure microvascular tone and permeability under precisely controlled chemical conditions and physical forces. The technical details and validation of the model are referenced by previous studies [3, 5-7, 15, 20, 21, 23]. We have taken the next step of answering questions regarding molecular mechanisms of these processes by specifically inhibiting key signaling proteins through peptide transfection of intact microvessels. An important advantage of this technique is that the effect of the signaling proteins can be immediately studied, without the need for transcription and translation required with DNA transfection. The latter not only requires long-term (24-48 h) incubation, but also produces proteins that may be structurally or functionally different from the one of interest. Additionally, our method of transfection is more conducive to this type of study than others, such as microinjection or electroporation, which would not be possible with intact vessels.

The results of our study demonstrate that arteriolar dilating response to bradykinin and serotonin is endothelium-dependent and NO-mediated. Transfection of a NOS-inhibitory peptide attenuated the vasodilatory responses to both bradykinin and serotonin in intact arterioles while having no effect on vasodilation induced by SNP. Because a vasodilatory response to SNP is endothelium-independent and elicited by NO activation of soluble guanylate cyclase in smooth muscle cells [24-27], this strongly suggests that our transfected peptide inhibited only eNOS. However, we do not exclude the possibility that the transfected protein may traverse the endothelium and enter the smooth muscle layer during prolonged periods of transfection. Experimental conditions should be closely monitored in future experiments to limit the cellular distribution of the transfected protein.

Venular hyperpermeability response to the PKC-activator PMA was attenuated to near control levels by transfection of a PKC-inhibitory peptide. We had previously shown this same peptide to inhibit PKC activity in coronary venular endothelial cells [18]. The transfection did not alter the increase in venular permeability elicited by the PKC-independent inflammatory factor histamine, further indicating the specific effect of the PKC-inhibiting peptide on venular barrier function. Upregulation of PKC and activation of NOS define two distinct signaling pathways involved in the regulation of microvascular function. While the elucidation of the complete pathways will require time and innovative studies, the results of this study strongly suggest that we can now more precisely study the roles of particular components of these pathways. Future studies will involve specific inhibition of other signaling and cytoskeletal proteins using inhibitory peptides or neutralizing antibodies.

\section{Acknowledgement}

This work was supported by National Heart, Lung, and Blood Institute Grant HL-61507. Sarah Y. Yuan is a recipient of National Institutes of Health Research Career Award K02 HL-03606. 


\section{References}

1 Davies PF: Flow-mediated endothelial mechanotransduction. Physiol Rev 1995;75:519560.

2 Hoek JB: Intracellular signal transduction and the control of endothelial permeability. Lab Invest 1992;67:1-4.

3 Huang Q, Yuan Y: Interaction of PKC and NOS in signal transduction of microvascular hyperpermeability. Am J Physiol 1997;273: H2442-H2451.

4 Lum H, Malik AB: Regulation of vascular endothelial barrier function. Am J Physiol 1994; 267:L223-L241.

5 Wu HM, Huang Q, Yuan Y, Granger HJ: VEGF induces NO-dependent hyperpermeability in coronary venules. Am J Physiol 1996; 271:H2735-H2739.

6 Yuan Y, Granger HJ, Zawieja DC, Chilian WM: Flow modulates coronary venular permeability by a nitric oxide-related mechanism. Am J Physiol 1992;263:H641-H646.

7 Yuan Y, Granger HJ, Zawieja DC, DeFily DV, Chilian WM: Histamine increases venular permeability via a phospholipase C-NO synthaseguanylate cyclase cascade. Am J Physiol 1993 264:H1734-H1739.

8 Yuan Y, Chilian WM: Heart microcirculation; in Barker JH, Anderson GL, Menger MD (eds): Clinically Applied Microcirculation Research. Boca Raton, CRC Press, 1995, pp 213-225.

9 Nishikawa Y, Stepp DW, Chilian WM: Nitric oxide exerts feedback inhibition on EDHFinduced coronary arteriolar dilation in vivo. Am J Physiol 2000;279:H459-H465.

10 Kajita Y, Takayasu M, Dietrich HH, Dacey RG Jr: Possible role of nitric oxide in autoregulatory response in rat intracerebral arterioles. Neurosurgery 1998;42:834-841.
11 Koller A, Huang A, Sun D, Kaley G: Exercise training augments flow-dependent dilation in rat skeletal muscle arterioles. Circ Res 1995;76: 544-550.

12 Shimamoto Y, Shimamoto H, Kwan CY, Daniel EE: Differential effects of putative protein kinase $\mathrm{C}$ inhibitors on contraction of rat aortic smooth muscle. Am J Physiol 1993;264: H1300-H1306.

13 Vuong PT, Malik AB, Nagpala PG, Lum H: Protein kinase $\mathrm{C}$ beta modulates thrombininduced $\mathrm{Ca}^{2+}$ signaling and endothelial permeability increase. J Cell Physiol 1998;175:379387.

14 Alexander JS, Jackson SA, Chaney E, Kevil $\mathrm{CG}$, Haselton FR: The role of cadherin endocytosis in endothelial barrier regulation: Involvement of protein kinase $\mathrm{C}$ and actin-cadherin interactions. Inflammation 1998;22:419-433.

15 Yuan Y, Huang Q, Wu HM: Myosin light chain phosphorylation: Modulation of basal and agonist-stimulated venular permeability. Am J Physiol 1997;272:H1437-H1443.

16 Tinsley $\mathrm{JH}, \mathrm{Wu} \mathrm{MH}$, Ma W, Taulman AC, Yuan SY: Activated neutrophils induce hyperpermeability and phosphorylation of adherens junction proteins in coronary venular endothelial cells. J Biol Chem 1999;274:24930-24934.

17 Tinsley JH, de Lanerolle P, Wilson E, Ma W, Yuan SY: Myosin light chain kinase transference induces myosin light chain activation and endothelial hyperpermeability. Am J Physiol 2000;279:C1285-C1289.

18 Tinsley JH, Hawker J, Yuan Y: Efficient protein transfection of cultured coronary venular endothelial cells. Am J Physiol 1998;275: H1873-H1878.

19 Bolz SS, Galle J, Derwand R, de Wit C, Pohl U: Oxidized LDL increases the sensitivity of the contractile apparatus in isolated resistance arteries for $\mathrm{Ca}^{2+}$ via a rho- and rho kinase-dependent mechanism. Circulation 2000;102:24022410 .
20 Yuan Y, Chilian WM, Granger HJ, Zawieja DC: Permeability to albumin in isolated coronary venules. Am J Physiol 1993;265:H543H552.

21 Yuan SY, Ustinova EE, Wu MH, Tinsley JH, $\mathrm{Xu}$ W, Korompai FL, Taulman AC: Protein kinase $\mathrm{C}$ activation contributes to microvascular barrier dysfunction in the heart at early stages of diabetes. Circ Res 2000;87:412-417.

22 House C, Kemp BE: Protein kinase C contains a pseudosubstrate prototype in its regulatory domain. Science 1987;238:1726-1728.

23 Huxley VH, Curry FE, Adamson RH: Quantitative fluorescence microscopy on single capillaries: $\alpha$-Lactalbumin transport. Am J Physiol 1987;252:H188-H197.

24 Mohazzab-H KM, Kaminski PM, Agarwal R, Wolin MS: Potential role of a membranebound NADH oxidoreductase in nitric oxide release and arterial relaxation to nitroprusside. Circ Res 1999;84:220-228.

25 Faraci FM, Sobey CG: Role of soluble guanylate cyclase in dilator responses of the cerebral microcirculation. Brain Res 1999;821:368373.

26 Tsuchida S, Hiraoka M, Sudo M, Kigoshi S, Muramatsu I: Attenuation of sodium nitroprusside responses after prolonged incubation of rat aorta with endotoxin. Am J Physiol 1994; 267:H2305-H2310.

27 Yousif MH, Oriowo MA, Williams KI: Sodium nitroprusside-induced cGMP-independent vasodilator responses in the perfused rabbit ovarian vascular bed. Pharmacol Res 1998;38:381386. 